\title{
1 Metabolomics and Systems Biology in Saccharomyces cerevisiae
}

\author{
J.I. Castrillo ${ }^{1}$, S.G. Oliver ${ }^{1}$
}

\section{CONTENTS}

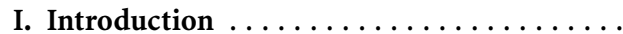

II. Saccharomyces cerevisiae: A Model Eukaryote and a Reference System in Biology .......

III. Functional Genomics of S. cerevisiae:

State of the Art..................

A. Functional Genomics: Levels of Regulation

B. S. cerevisiae Functional Genomics:

State of the Art ................

IV. Metabolomics in Comprehensive

Post-Genomic Studies: Towards Systems

Biology Using S. cerevisiae as a Model .....

A. Metabolomic Studies: Metabolic Networks and Participation of Metabolites in Regulation ................

B. Integrative Studies for Global Description of Cellular Complexity: Cellular Networks and Systems Biology..............

C. S. cerevisiae as a Reference Model in Systems Biology: Advanced Studies, Biological Networks and Genome-Scale Models, and Applications . . . . . . . . . .

V. Conclusions ..................

References .....................

\section{Introduction}

The genomic revolution has been characterized by the generation of huge amounts of information in the form of genome sequences, and of their direct application in comprehensive molecular studies and comparative genomics strategies (e.g. Goffeau et al. 1996, 1997; The International Human Genome Mapping Consortium 2001a, 2001b; von Mering et al. 2002; Gavin and Superti-Furga 2003). In the post-genomic era, the explosion of new technologies, whose exploitation is becoming progressively more refined, is facilitating the study of biological systems on a genome-wide scale. These studies are being performed at different functional genomic levels, including the genome, transcriptome, proteome and metabolome, and in a progressively

${ }^{1}$ Faculty of Life Sciences, Michael Smith Building, The University of Manchester, Oxford Road, Manchester M13 9PT, UK more integrative way (Oliver 1997, 2002; Oliver et al. 1998; ter Linde et al. 1999; Delneri et al. 2001; Castrillo and Oliver 2004 and references therein).

At the same time that these techniques are being applied and refined, the complexity of biological systems is being rediscovered. Living things are made up of thousands of components (genes, transcripts, proteins and metabolites), which are subject to modification by post-transcriptional and post-translational mechanisms. These components participate in anabolic, catabolic and regulatory networks in response to environmental and developmental signals, many of which are still to be elucidated (Castrillo and Oliver 2004 and references therein). In this context, the utilization of welldefined model systems under controlled conditions is of central importance in the drive towards an integrative systems biology perspective of the cell.

The purpose of this chapter is to present an up-to-date view of the functional genomics of Saccharomyces cerevisiae and its growing potential as a model system for systems biology studies. The importance of including metabolomics as part of this integrative approach to the study of the eukaryotic cell as a biological system is emphasised.

\section{Saccharomyces cerevisiae: A Model Eukaryote and a Reference System in Biology}

Saccharomyces cerevisiae is a species of budding yeast, a group of unicellular fungi belonging to the phylum Ascomycetes. S. cerevisiae is being used as a model eukaryote in biology because the basic mechanisms of DNA replication, chromosomal recombination, cell division, gene expression, and metabolism are generally conserved between yeast and higher eukaryotes (i.e. mammals; Rose and Harrison 1987-1995; Sherman 1998, 2002; Castrillo and Oliver 2004). 
Among the properties which make S. cerevisiae a particularly suitable organism for biological studies are its Generally Regarded As Safe (GRAS) status, rapid growth, well-dispersed cells, simple methods of cultivation under controlled conditions, ease of replica plating and mutant isolation. Moreover, yeasts represent a well-defined genetic system with facile techniques of genetic manipulation (Brown and Tuite 1998; Sherman 1998, 2002; Castrillo and Oliver 2004).

The favourable characteristics of this yeast, together with its easy accessibility due to its economic importance in beer- and bread-making, make it a cheap source for biochemical studies. Thus, many metabolites and enzymes of central metabolic processes (i.e. Embden-Meyerhoff pathway, tricarboxylic acid cycle, TCA), and the first complete map of central metabolic pathways were first unveiled in $S$. cerevisiae, which is used as a touchstone model for the study of the eukaryotic cell (Lehninger 1975; Rose and Harrison 1987-1995; Fell 1997; Alberts et al. 2002; Castrillo and Oliver 2004 and references therein). As a result of all this, a wide knowledge of the genetics, biochemistry and physiology of this yeast is presently available (Rose and Harrison 1987-1995; Brown and Tuite 1998; Sherman 1998, 2002; Burke et al. 2000; Guthrie and Fink 2002a, 2002b, 2004).

At the same time as being considered a platform for basic studies, S. cerevisiae has attracted considerable interest from the early days of microbiology as a 'biological system', capable of performing specific biotransformations of interest to the fermentation industry (Pasteur 1857; Rose and Harrison 1993; Olson and Nielsen 2000; Schwartz 2001; Ton and Rao 2004). As a consequence of this, $S$. cerevisiae cultures have been subjected to a number of modelling strategies directed towards the representation of different metabolic and cell biological processes. These were simple models at first, with limited information on the metabolism and internal regulatory mechanisms, i.e. basic unstructured models, in which cell growth was modelled as an autocatalytic process, and the specific rates of individual reactions were based on kinetic models (e.g. Monod; cf. Bailey and Ollis 1986; Sinclair and Cantero 1990). As a more complete knowledge of the metabolic pathways and control mechanisms became available, more structured models and comprehensive modelling strategies (i.e. metabolic steady-state flux models including information on yeast central metabolic pathways and cybernetic models) could be applied (Bailey and Ollis 1986; Castrillo and Ugalde 1994 and references therein; Cortassa and Aon 1994; Giuseppin and van Riel 2000; Lei et al. 2001). At this point, attempts to increase the flux through specific pathways by metabolic engineering techniques (Bailey 1991; Stephanopoulos and Vallino 1991) met with only limited success, revealing our lack of real understanding of the dynamics of metabolism in S. cerevisiae. Such an outcome was anticipated (at least, by some) as a direct consequence of the application of the Metabolic Control Analysis (MCA) theory, a fundamental framework in quantitative modelling and metabolic control (Kacser and Burns 1973; Kacser 1995; Fell 1997; see also last section).

If $S$. cerevisiae is to fulfil its potential as a model eukaryote, and as a reference 'biological system' at the cellular level, the progressive incorporation of functional genomic information at the different levels (i.e. genome, transcriptome, proteome and metabolome) into mathematical models representative of the global behaviour of the cell will be required (Kitano 2002; Ideker 2004a). S. cerevisiae was the first eukaryotic organism for which the complete genome was sequenced (Goffeau et al. $1996,1997)$. Hence, it is at the forefront of the postgenomic era (Castrillo and Oliver 2004; see next section).

\section{Functional Genomics of $S$. cerevisiae: State of the Art}

\section{A. Functional Genomics: Levels of Regulation}

A schematic representation of the eukaryotic cell as a global system, with the different levels of functional genomics (genome, transcriptome, proteome and metabolome; Oliver 1997, 2002; Oliver et al. 1998; Delneri et al. 2001), their localization and interactions, main regulatory circuits and relationships is presented in Fig. 1.1. Our current view of the eukaryotic cell is that of a system characterized by a coordinate integration of the different functional genomic levels and individual networks, in direct relation with the environment. This system is intrinsically complex and involves the integration of regulatory mechanisms at the genomic, transcriptional, posttranscriptional, post-translational and metabolic 


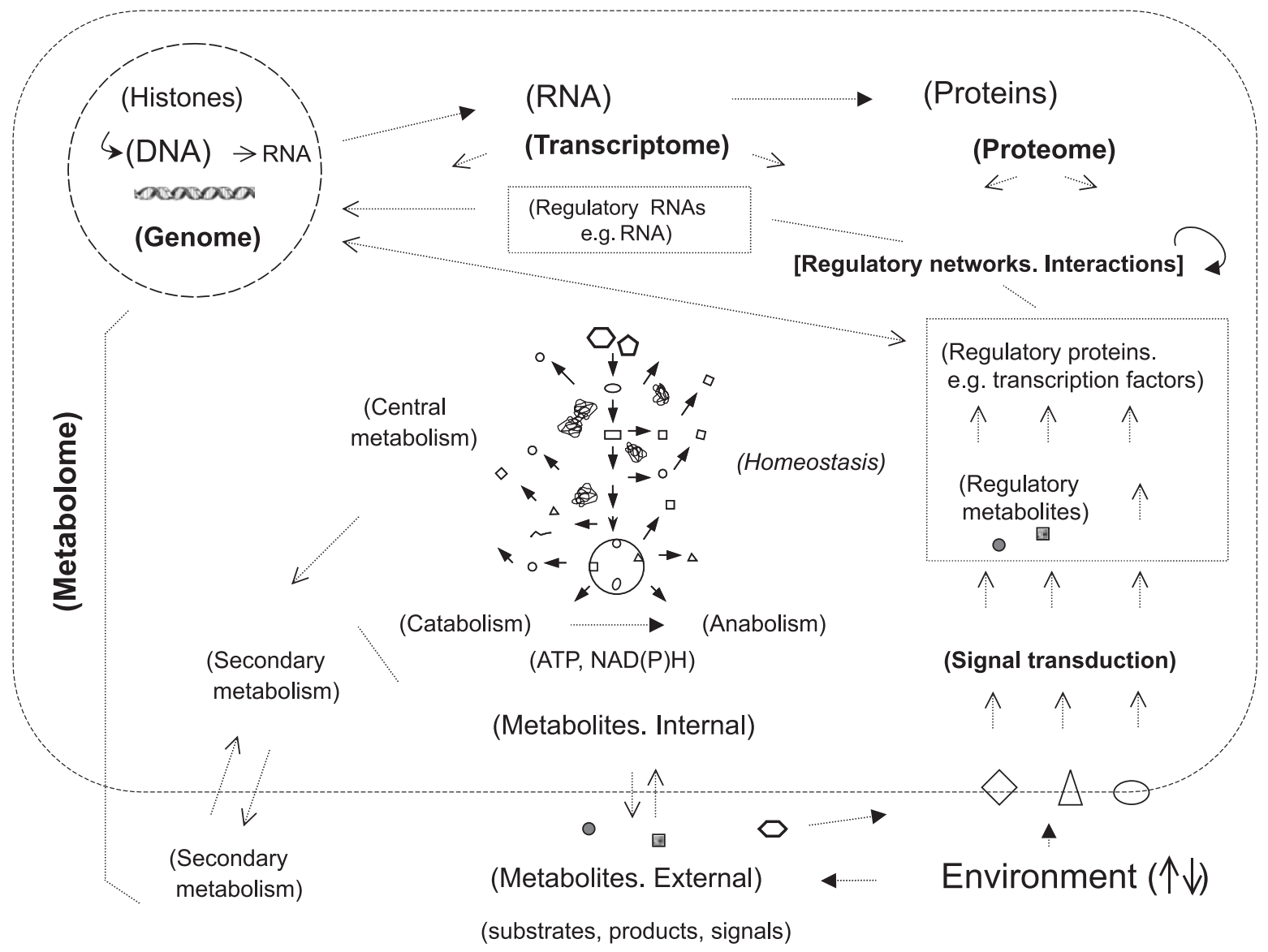

Fig. 1.1. Eukaryotic cell. Functional genomic levels and regulatory mechanisms

levels (Fafournoux et al. 2000; Muratani and Tansey 2003; Verger et al. 2003; Castrillo and Oliver 2004; Choudhuri 2004). Among the most relevant mechanisms are epigenetic mechanisms (e.g. DNA methylation, histone modifications), mRNA splicing and small regulatory RNAs, protein methylation, glycosylation, ubiquitination and sumoylation, protein-protein and proteinmetabolite interactions, and participation of metabolites together with transcription factors and regulatory proteins in signal transduction pathways (Day and Tuite 1998; Castrillo and Oliver 2004 and references therein; Choudhuri 2004).

\section{B. S. cerevisiae Functional Genomics: State of the Art}

S. cerevisiae (laboratory strain S288C; MAT $\alpha$ SUC2 mal mel gal2 CUP1 flo1 flo8-1 hap1; Mortimer and Johnston 1986; Gaisne et al. 1999; Sher- man 2002) was the first eukaryotic organism for which the whole genome sequence was completed and made publicly available (Goffeau et al. 1996, 1997). This sequence has been further certified as the best annotated eukaryotic genome (Goffeau 2000). The collection of complete $S$. cerevisiae chromosome sequences and annotations is available at Goffeau et al. (1997, http://www.nature.com/genomics/papers/ s_cerevisiae.html), and can also be obtained at the National Centre for Biotechnological Information (NCBI; http://www.ncbi.nlm.nih.gov/mapview/ map_search. cgi? taxid=4932). In this case, the nucleotide and protein sequences are provided by the Saccharomyces Genome Database (SGD; http://www.yeastgenome.org) and are revised as SGD is updated.

The S. cerevisiae genome contains about $12 \mathrm{Mb}$ of DNA distributed between 16 chromosomes which contain a total of about 6000 genes, with a relatively low proportion containing introns 
Table.1.1. Characteristics of Saccharomyces cerevisiae (Goffeau et al. 1996; Sherman 1998, 2002; National Cen- tre for Biotechnological Information, NCBI, http://www. ncbi.nlm.nih.gov/mapview/map_search.cgi?taxid=4932)

\begin{tabular}{lll}
\hline S. cerevisiae & Characteristics & \\
\hline Lineage & Eukaryota; Fungi; Ascomycota; Saccharomycotina; \\
& Saccharomycetes; Saccharomycetales; Saccharomycetaceae; \\
& Saccharomyces & \\
Nuclear genome & 16 chromosomes $(12,052 \mathrm{~Kb})$ & \\
& $2 \mu$ m circle plasmid $(6.3 \mathrm{~Kb})$ \\
Mitochondrial genome & Mitochondrial DNA $(850 \mathrm{~Kb})$ \\
ORFs & 6183 ORFs encoding for 5773 proteins \\
tRNA genes & 262 tRNA genes & \\
Introns & $3.8 \%$ & \\
Intracellular dsRNA viruses & $0.1 \%$ of total nucleic acid content & \\
Cells & Haploid & Diploid \\
Volume per cell $\left(\mu \mathrm{m}^{3}\right)$ & 70 & 120 \\
Global content & 1 mg wet weight or 0.25 mg & 1 mg wet weight or 0.25 mg \\
& dry weight contains & dry weight contains \\
& $\sim 0.28 \mu$ of DNA & $\sim .42 \mu$ of DNA \\
& $20 \mu g$ of total RNA & $24 \mu$ of total RNA \\
& $\sim 1 \mu$ of mRNA & $\sim 1.2 \mu$ of mRNA \\
& 0.10 mg total protein & 0.10 mg total protein \\
\hline
\end{tabular}

( 4\%; Goffeau et al. 1996, 1997; Brown and Tuite 1998; Sherman 1998, 2002). From the whole genome, a total of 5257 protein-coding genes have been annotated (i.e. reported to code for a specific protein with a gene ontology category curated with experimental evidence) as of the end of October 2004 (Yeast Proteome Database, http://proteome.incyte.com; Costanzo et al. 2001; Csank et al. 2002). The main characteristics of $S$. cerevisiae are summarized in Table 1.1.

Once the genome sequence is known, postgenomic studies entail, first, the design and implementation of advanced high-throughput methods and genomic strategies to extract the maximum information at the different functional genomic levels and, second, an efficient analysis of the huge amount of data generated, in order to extract valid conclusions and new knowledge (e.g. mechanisms of regulation and their integration). The most advanced functional genomics methods and strategies investigated in S. cerevisiae have been reviewed by Castrillo and Oliver (2004). A summary of the main databases and resources for analysis of yeast genomic data is presented in Table 1.2. The most relevant strategies are:

1. At the genome level, application of molecular genetic techniques on a large scale for the generation of comprehensive collections of yeast mutants. Thus, for example, single deletion mutants, double mutants and the 'TRIPLES' collection of mutants, obtained by random transposon insertion, are used for global analyses, functional profiling and gene characterization (Ross-Macdonald et al. 1999; Winzeler et al. 1999; Giaever et al. 2002; Scherens and Goffeau 2004 and references therein). To complement yeast knockout collections, the construction of a collection of yeast strains in which a tetracycline-responsive promoter is inserted upstream of individual essential genes will allow us to explore the function of essential genes via conditional and titratable promoter alleles (Eisenstein 2004; Mnaimneh et al. 2004).

2. At the gene expression (transcriptome) level, microarrays have been widely used for the global analysis of yeast gene expression patterns (Lashkari et al. 1997; Wodicka et al. 1997; Spellman et al. 1998), with more recent studies revealing the importance of a careful experimental design, controlled conditions and good strategies for data processing and statistical analysis (ter Linde et al. 1999; Hayes et al. 2002; Boer et al. 2003; Tilstone 2003). In addition, new approaches to analyse not only relative changes in gene expression (mRNA) levels but also net transcription rates on a genomic scale are progressively being incorporated (Iyer and Struhl 1996; Hirayoshi and Lis 1999; Garcia-Martinez et al. 2004).

3. At the proteome level: the first whole-proteome microarray was developed for yeast, and advanced strategies for the preparation of such 
Table. 1.2. Main resources, tools and databases for individual and integrative analysis of yeast genomics data

\begin{tabular}{|c|c|}
\hline Databases/resources & Reference \\
\hline \multicolumn{2}{|l|}{ Genome databases } \\
\hline S. cerevisiae Genome Database (SGD) & http://www.yeastgenome.org \\
\hline National Centre for Biotechnological Information & http://www.ncbi.nlm.nih.gov/mapview/map_search.cgi?taxid=4932 \\
\hline $\begin{array}{l}\text { Munich Information centre } \\
\text { for Protein Sequences (MIPS) }\end{array}$ & http://mips.gsf.de/genre/proj/yeast/index.jsp \\
\hline S. cerevisiae mutants collection & http://www.uni-frankfurt.de/fb15/mikro/euroscarf/complete.html \\
\hline S. cerevisiae resource center & http://depts.washington.edu/ yeastrc \\
\hline \multicolumn{2}{|l|}{ Gene expression (transcriptome) resources } \\
\hline Microarray standards (MIAME) & http://www.mged.org/miame \\
\hline ArrayExpress & http://www.ebi.ac.uk/arrayexpress \\
\hline Stanford Microarray Database & http://genome-www5.stanford.edu \\
\hline \multicolumn{2}{|c|}{ Promoter, regulatory sequences and transcription factor databases } \\
\hline S. cerevisiae promoter database & http://cgsigma.cshl.org/jian/ \\
\hline $\begin{array}{l}\text { Yeast Transcription Factors } \\
\text { and related components (YTF) }\end{array}$ & http://biochemie.web.med.uni-muenchen.de/YTFD/ \\
\hline TRANSFAC & http://www.biobase.de/pages/products/transfac.html \\
\hline \multicolumn{2}{|l|}{ Proteome resources } \\
\hline Proteomics Standards Initiative (PSI) & http://psidev.sourceforge.net \\
\hline Proteomics platform (PEDRo) & http://pedro.man.ac.uk/ \\
\hline Protein-protein interactions & (von Mering et al. 2002) \\
\hline Yeast proteins localization & http://yeastgfp.ucsf.edu/ \\
\hline Yeast protein microarrays & (Zhu and Snyder 2003) \\
\hline \multicolumn{2}{|l|}{ Metabolic pathways databases } \\
\hline KEGG & http://www.genome.ad.jp/kegg/ \\
\hline BioCyC & http://biocyc.org \\
\hline PathDB & http://www.ncgr.org/pathdb/ \\
\hline RIKEN & http://genome.gsc.riken.go.jp/DNA-Book/metabolome.shtml \\
\hline Biomolecular interactions, BIND & http://www.blueprint.org/bind/bind.php \\
\hline \multicolumn{2}{|c|}{ Tools for analysis and management of global genomic information } \\
\hline Gene Ontology tools & http://www.geneontology.org/GO.tools.shtml \\
\hline - GoMiner & http://discover.nci.nih.gov/gominer/ \\
\hline - GenMAPP & http://www.genmapp.org/ \\
\hline Genome Information Management & http://www.cs.man.ac.uk/img/gims/ (Cornell et al. 2003) \\
\hline \multicolumn{2}{|l|}{ System (GIMS) } \\
\hline \multicolumn{2}{|l|}{ Systems biology resources } \\
\hline Yeast Systems Biology Network & http://www.ysbn.org \\
\hline Systems Biology DataBase (SBDB) & http://www.sysbioldb.org \\
\hline
\end{tabular}

arrays have been developed (Zhu et al. 2001, 2003; Zhu and Snyder 2003). In addition, studies on sub-cellular localization of yeast proteins on a proteome-wide scale, phosphoproteome studies, protein-protein interaction maps and studies on protein turnover have all been undertaken (Ficarro et al. 2002; Gavin et al. 2002; Ho et al. 2002; von Mering et al. 2002; Pratt et al. 2002; Ghaemmaghami et al. 2003; Huh et al. 2003; Wohlschlegel and Yates 2003).

4. At the metabolome level, new methods for the analysis of yeast metabolites, strategies to ascribe function to unknown genes, and the classification of yeast mutants using metabolic fingerprinting and footprinting have been devel- oped (Gonzalez et al. 1997; Raamsdonk et al. 2001; Allen et al. 2003; Castrillo et al. 2003).

5. At the bioinformatics level, new machinelearning methods for the analysis of transcriptome, proteome and metabolome data, and for the study of yeast regulatory networks have been derived. Relevant web resources, databases and methods for the global analysis of yeast genomic data, data repositories and data warehouses for storage and rapid access to yeast genomic raw data have all been generated. These include the Saccharomyces Genome Database (SGD, http://www.yeastgenome.org), microarray databases and repositories (e.g. Stanford Microarray Database and Array- 